\title{
The Fermi accelerator in atom optics
}

\author{
Farhan Sai由, Iwo Bialynicki-Birula**, Mauro Fortunatol and Wolfgang P. Schleich \\ Abteilung für Quantenphysik, Universität Ulm, Albert-Einstein-Allee 11, D-89069 Ulm, Germany
}

\begin{abstract}
We study the classical and quantum dynamics of a Fermi accelerator realized by an atom bouncing off a modulated atomic mirror. We find that in a window of the modulation amplitude dynamical localization occurs in both position and momentum. A recent experiment [A. Steane, P. Szriftgiser, P. Desbiolles, and J. Dalibard, Phys. Rev. Lett. 74, 4972 (1995)] shows that this system can be implemented experimentally.
\end{abstract}

PACS numbers: 72.15.Rn, 47.52.+j, 03.75, 03.65.-w

Almost fifty years ago Enrico Fermi [1] suggested that it is collisions with moving magnetic fields that accelerate cosmic rays. Since then many models associated with the name of Fermi accelerator describing particles colliding with moving walls have been investigated theoretically [2] 6]. The recent experiment [7] on atoms bouncing off a modulated atomic mirror constitutes an atom optics [8] version of the Fermi accelerator. In the present paper, we argue that this system serves as a testing ground for classical and quantum chaos [9]. We show that there exists an experimentally accessible parameter regime in which such experiments can bring out many features of the Fermi accelerator.

Three properties make this model rather special [10] in the field of chaos: (i) It is one of the very few bound systems with no continuum 11, 12], (ii) the phenomenon of dynamical localization occurs in the position and momentum variables [13], (iii) dynamical localization arises only in a window of the modulation depth; the onset of classical chaos sets the lower boundary, whereas the effective dimensionless Planck constant $k$ determines the upper boundary. Therefore, we can tune the system continuously from a regime of no chaos, through one with classical chaos but dynamical localization, to one with classical chaos and no dynamical localization. The experiments performed so far have focused on the latter regime. However, we show that they can easily be extended into the localization window.

We now consider a cloud of laser-cooled atoms stored in a magneto-optical trap. When we switch off the trap, the atoms move along the $\tilde{z}$-direction [14] under the influence of gravity and bounce off an atomic mirror 15. The latter results from a laser field incident on a glass prism under an angle of total internal reflection. This creates an evanescent wave whose intensity $I(\tilde{z})=I_{0} \exp (-2 k \tilde{z})$ decays over a distance $k^{-1}$ outside of the prism. A sinusoidal modulation [7] of the intensity with amplitude $\epsilon$ and frequency $\omega$ changes the intensity $I(\tilde{z})$ to $I(\tilde{z}, \tilde{t})=I_{0} \exp (-2 k \tilde{z})(1+\epsilon \sin \omega \tilde{t})$. In our calculations we have used a slightly different form

$$
I(\tilde{z}, \tilde{t})=I_{0} \exp (-2 k \tilde{z}+\epsilon \sin \omega \tilde{t})
$$

of modulation which corresponds to an oscillation of the mirror and is our exponential model of the Fermi accelerator. When $\epsilon$ is not too large, the results do not depend significantly on the form of the modulation.

In order to avoid problems associated with spontaneous emission we consider a large detuning between the laser light field and the atomic transition frequency. This ensures that the atom rarely leaves the ground state. The dynamics of the center-of-mass motion of the atom in the ground state follows then from the Hamiltonian

$$
H=\frac{\tilde{p}^{2}}{2 m}+m g \tilde{z}+\frac{\hbar \Omega_{e f f}}{4} e^{-2 k \tilde{z}+\epsilon \sin \omega \tilde{t}} .
$$

Here $\tilde{p}$ is the momentum of the atom of mass $m$ along the $\tilde{z}$-axis, and $g$ denotes the gravitational acceleration.

We introduce the dimensionless position and momentum coordinates $z \equiv \tilde{z} \omega^{2} / g$ and $p \equiv \tilde{p} \omega /(m g)$ and time $t \equiv \omega \tilde{t}$ together with the dimensionless intensity $V_{0} \equiv \hbar \omega^{2} \Omega_{e f f} /\left(4 m g^{2}\right)$, steepness $\kappa \equiv 2 k g / \omega^{2}$ and the modulation depth $\lambda \equiv \omega^{2} \epsilon /(2 \mathrm{~kg})$ of the evanescent wave. In these variables, the classical dynamics follows from the Hamilton equations of motion,

$$
\begin{aligned}
& \dot{z}=p, \\
& \dot{p}=-1+\kappa V_{0} \exp [-\kappa(z-\lambda \sin t)] .
\end{aligned}
$$

The corresponding Schrödinger equation for the atoms in the ground state reads

$$
i \hbar \frac{\partial \psi}{\partial t}=\left[\frac{p^{2}}{2}+z+V_{0} \exp [-\kappa(z-\lambda \sin t)]\right] \psi,
$$

where $\hbar \equiv \hbar \omega^{3} /\left(m g^{2}\right)$ denotes the dimensionless Planck constant 16].

In this paper, we use Eqs. (3) and (位) to determine the classical and quantum mechanical position and momentum distributions of cold atoms bouncing under the influence of gravity off an oscillating mirror. In all our calculations we start at $t=0$ from an ensemble of atoms with an average momentum zero and an average position $z=20$ above the mirror. We find dynamical localization in the quantum case. However, localization occurs only over a certain range of the modulation depth $\lambda$. In order to understand this result and to find the lower and upper 
boundaries $\lambda_{l}$ and $\lambda_{u}$ of this regime, we approximate the exponential potential by a hard wall, that is an infinitely steep and infinitely high wall and borrow some results [5,6, 17] for such a time dependent triangular potential well.

We can approximate the dynamics of this system by a map [2] connecting two consecutive bounces of the atom: The momentum $p$ of the atom and the phase $\theta$ of the wall before a bounce determine the momentum $\bar{p}$ and phase $\bar{\theta}$ just before the next bounce through the standard Chirikov-Taylor map

$$
\begin{aligned}
& \bar{p}=p+K \cos \theta \\
& \bar{\theta}=\theta+\bar{p} \quad \bmod (2 \pi)
\end{aligned}
$$

with $K=4 \lambda$.

Chirikov 18] has shown that when the chaos parameter $K$ becomes larger than the critical value $K_{c r}=0.9716 \ldots$, the classical system undergoes a global diffusion. This implies for our driven triangular potential well that diffusive dynamics sets in for $\lambda>\lambda_{l}=K_{c r} / 4 \approx 0.24$. Below this value we have isolated resonances, whereas above, the resonances overlap and we have islands embedded in a stochastic sea.

In the corresponding quantum mechanical system, the quasi-energy spectrum of the Floquet operator changes from a point spectrum to an almost continuum [6] when $\lambda>\lambda_{u} \equiv \sqrt{\hbar} / 2$. Here quantum diffusion destroys localization. The conditions of classical and quantum diffusion, together, define the window

$$
0.24<\lambda<\frac{\sqrt{\hbar}}{2}
$$

in the modulation strength, where we can find dynamical localization.

This result obtained for the triangular well determines an approximate range of $\lambda$ in which we can observe dynamical localization for the exponential well. Since the lower boundary $\lambda_{l}$ is set by classical dynamics we can find $\lambda_{l}$ by evaluating Lyapunov exponents. For a modulation amplitude $\lambda<0.24$, the Lyapunov exponent converges to zero in a vast range of initial conditions except in small regions near separatrices as shown in Fig. 11(a). However, for larger modulations diffusion occurs and the Lyapunov exponent becomes positive in a vast range of initial conditions as shown in Fig. 11(b).

Above the lower boundary, the classical system undergoes diffusion in both position and momentum space. This diffusion manifests itself in a linear growth of the square $\Delta p^{2}$ of the width $\Delta p \equiv\left(\left\langle p^{2}\right\rangle-\langle p\rangle^{2}\right)^{1 / 2}$ of the classical momentum distribution and of the width $\Delta z \equiv$ $\left(\left\langle z^{2}\right\rangle-\langle z\rangle^{2}\right)^{1 / 2}$ of the classical position distribution with time as shown in Fig. 2. The linear growth of $\Delta p^{2}$ with time, $\Delta p^{2} \sim D t$, also follows from the Chirikov-Taylor map [5] of Eq. (5). However, the value of the diffusion constant obtained for our choice of parameters is much smaller than the value $D=K^{2} / 2$ predicted by the Chirikov-Taylor map.

We note that the numerical result of Fig. 2 also suggests a linear time dependence of the width $\Delta z$ of the position distribution. In addition we see that the values of $\Delta p^{2}$ and $\Delta z$ are approximately equal. These facts can be explained by assuming that the distribution of positions and momenta is governed by the classical Boltzmann distribution

$$
P_{c l}(z, p)=(2 \pi)^{-1 / 2} \eta^{-3 / 2} \exp \left[-\left(p^{2} / 2+V(z)\right) / \eta\right]
$$

where

$$
V(z)=z+V_{0} \exp (-\kappa z)
$$

Here, the quantity $\eta$ depends on time and plays the role of an effective temperature. This conjecture is supported by the fact that the Boltzmann statistics holds in case of diffusive dynamics [19].

The calculation of the $\Delta z$ and $\Delta p^{2}$ can be performed analytically in the simplest case of the triangular potential well and it gives the equality

$$
\Delta z=\eta=\Delta p^{2}
$$

We have checked that for an exponential barrier this equality is also approximately true.

We also show in Fig. 3 the average momentum $\bar{p}$ and average position $\bar{z}$. Classically, the average momentum oscillates around zero, which corresponds to our initial average momentum, in agreement with the result obtained from Chirikov map. On the other hand, the average position displays a linear rise with time, in accordance with the relation calculated with the Boltzmann distribution.

The classical position and momentum distributions shown in Fig. 4 follow then the exponential barometric formula $P_{c l}(z)=\eta^{-1} \exp (-z / \eta)$ and the Gaussian distribution $P_{c l}(p)=(2 \pi \eta)^{-1 / 2} \exp \left[-p^{2} /(2 \eta)\right]$ predicted from Eq. (7).

With the help of these distributions we can easily establish the relation $\Delta z=\eta=\Delta p^{2}$. Since we have the diffusion law $\Delta p^{2} \sim D t$, Eq. (9) predicts that $\Delta z \sim D t$ in agreement with the numerical results of Fig. 2 .

In contrast to this classical diffusion the corresponding quantum mechanical quantities saturate after an initial rise that is of classical nature. This difference between classical and quantum dynamics manifests itself after the quantum break time $t^{*}$. We estimate [9] this time as $t^{*} \sim D k^{-2}$ and the corresponding saturation value of $\Delta p^{2}$ follows from $\Delta p^{2} \sim D t^{*} \sim D^{2} k^{-2}$.

We also note characteristic oscillations in the quantum mechanical widths $\Delta p^{2}$ and $\Delta z$. These oscillations, absent in the classical curves, are a generalization to driven systems [21] of the revival phenomena 20]. 
The quantum distributions in position and momentum shown in Fig. 1 are completely different from their classical counterparts. Indeed the quantum mechanical momentum distribution is exponential rather than Gaussian. Moreover, the quantum mechanical position distribution contains two exponentials: The steep one corresponds to dynamical localization in position whereas the flat one has the same steepness as the classical barometric formula. This separation of the quantum distribution into a quantum and a classical part also occurs in other bound problems 12 .

A qualitative comparison between the quantum triangular well and the kicked rotator model [9] yields the localization length as $l \sim D k^{-2} \sim 8 \lambda^{2} / k^{2}$. In our numerical investigations we clearly see this qualitative dependence on $k$ and $\lambda$.

The initial minimum uncertainty wavepacket delocalizes when the modulation depth exceeds the upper boundary $\lambda_{u}$ of the localization window. In Fig. 5 we show the widths of the classical (thick line) and the quantum mechanical (thin line) momentum distribution as a function of the modulation amplitude $\lambda$. We find exponential localization within the window, as shown in the inset (a). However, above the upper boundary $\lambda_{u}$, quantum diffusion sets in resulting in the Gaussian distribution shown in the inset (b).

We now make contact with the recent experiment [7] on the phase modulation of de Broglie waves. In this experiment cesium atoms bounce off a mirror with $k^{-1}=0.19 \mu \mathrm{m}$. The mirror is modulated with an amplitude $\epsilon=0.82$ and various frequencies of the order $\omega \approx 2 \pi \times 900 \mathrm{kHz}$. With the mass $m=2.21 \times 10^{-25} \mathrm{~kg}$ of Cs atoms, the gravitational acceleration $g=9.81 \mathrm{~m} / \mathrm{s}^{2}$, and $\hbar=6.673 \times 10^{-34} / 2 \pi \mathrm{Js}$, we find the dimensionless Planck constant $\hbar \approx 9 \times 10^{8}$ and the modulation depth $\lambda \approx 2.5 \times 10^{5}$. We emphasize that this value is larger than $\lambda_{u}=\sqrt{\hbar} / 2 \sim 1.5 \times 10^{4}$. Therefore this experiment lies outside of the localization window.

However, a modulation frequency of $\omega=2 \pi \times$ $1.477 \mathrm{kHz}$, the decay length $k^{-1}=0.455 \mu \mathrm{m}$ and the effective Rabi frequency $\Omega_{\text {eff }}=2 \pi \times 88.8 \mathrm{kHz}$ lead to $\hbar=4, \kappa=0.5$ and $V_{0}=60$ which are the values used throughout our paper. We have chosen this value of $V_{0}$ to guarantee that the atoms will not hit the surface of the mirror which is situated at $z=0$. Of course some atoms have enough energy to break through the barrier but their number is negligible. The lower and upper boundary $\lambda_{l}=0.24$ and $\lambda_{u}=1$ then translate into an intensity modulation of $\epsilon_{l}=0.12$ and $\epsilon_{u}=0.5$, respectively.

With our parameters we have observed dynamical localization within 100 bounces. The quantum break time $t^{*} \sim 75 \mathrm{~ms}$ corresponds to 50 bounces. Since Ref. [22] reports more than hundred bounces, this effect should be observable.

Note that the initial condition in [7] was $\tilde{z}_{0}=3.3 \mathrm{~mm}$ which corresponds to $z_{0}=2.9 \times 10^{4}$ in dimensionless coordinates. In contrast, in our numerical calculations we have chosen $z_{0}=20$ which corresponds to $\tilde{z}=2.27 \mu \mathrm{m}$. However, a recent experiment 22 shows that even this is possible. We are aware of the fact that the practical implementation of our proposed experiment may not be trivial. However, in spite of the experimental challengedue to the external modulation of the mirror-we are confident that an experiment along the above lines can be performed.

We conclude by summarizing our main results. An atom bouncing off a modulated mirror under the influence of gravity exhibits dynamical localization in position and momentum. However, this effect only occurs in a window of modulation. Our investigations show that bound systems may exhibit a rich dynamical behavior both in classical and quantum domain, which is substantially different from the standard kicked rotator model. These conclusions motivate further theoretical and experimental studies of bound systems from the view point of chaos. Since our system in the absence of the driving force does not contain any continuum of states it is cleaner than the microwave driven hydrogen atom. Currently available experimental technology allows us to observe this phenomenon.

Our analysis of the atomic Fermi accelerator is based on a laser mirror for the atom and therefore makes use of the interaction of the atom with an evanescent laser field. However, it is interesting to note that also magnetic mirrors 23] for atoms exist and have produced many bounces. A modulation of such a magnetic mirror could offer another possible realization of the atomic Fermi accelerator.

We thank G. Alber, M. El Ghafar, R. Grimm, E. Mayr, P. Törmä, M. G. Raizen, V. Savichev and A. Zeiler for many fruitful discussions. One of us FS thanks the Ministry of Science and Technology, Pakistan for its continuous support. IBB is grateful to the Humboldt Foundation and $\mathrm{MF}$ thanks the European Community for a grant in the framework of the HCM network "Non-Classical Light" and INFM. This work was partially supported by DFG.

* E-mail address: saif@physik.uni-ulm.de

** Permanent address: Center for Theoretical Physics, Lotników 32/46, 02-668 Warsaw, Poland.

$\dagger$ Permanent address: Istituto Nazionale per la Fisica della Materia, Università di Camerino, Dipartimento di Matematica e Fisica, via Madonna delle Carceri, 62032 Camerino, Italy.

[1] E. Fermi, Phys. Rev. 75, 1169 (1949).

[2] A.J. Lichtenberg and M.A. Lieberman, Regular and 
Stochastic Motion, (Springer, Berlin, 1983) and references therein.

[3] Wen-Yu Chen and G. J. Milburn, Phys. Rev. E 56, 351 (1997).

[4] M. Holthaus and M. E. Flatté, Phys. Lett. A 181, 151 (1994).

[5] F. Benvenuto, G. Casati, I. Guarneri and D.L. Shepelyansky, Z. Phys. B 84, 159 (1991).

[6] C. R. de Oliveira, I. Guarneri and G. Casati, Europhys. Lett. 27, 187 (1994).

[7] A. Steane, P. Szriftgiser, P. Desbiolles and J. Dalibard, Phys. Rev. Lett. 74, 4972 (1995).

[8] For a review of the field of atom optics see the special issues, Appl. Phys. B 54, 319 (1992); J. Phys. II 4, 1877 (1994); Quantum Semicl. Opt. 8, 495 (1996).

[9] F. Haake, Quantum Signatures of Chaos, (Springer, Berlin 1992).

[10] The field of atom optics as a testing ground for quantum chaos started with the theoretical proposal of R. Graham, M. Schlautmann and P. Zoller, Phys. Rev. A 45, R19 (1992) followed by the experiment of F. L. Moore, J. C. Robinson, C. Bharucha, P. E. William and M. G. Raizen, Phys. Rev. Lett. 73, 2974 (1994). For review see M. G. Raizen, in Advances in Atomic, Molecular, and Optical Physics Vol. 41, p. 41 ed. B. Bederson and H. Walther (Academic Press, New York 1999).

[11] For the microwave driven hydrogen atom the continuum plays an important role. See for example, F. Benvenuto, G. Casati and D. L. Shepelyansky, Phys. Rev. A 55, 1732 (1997); M. Arndt, A. Buchleitner, R. N. Mantegna and H. Walther, Phys. Rev. Lett. 67, 2435 (1991); J. E. Bayfield, G. Casati, I. Guarneri and D.W. Sokol, Phys. Rev. Lett. 63, 364 (1989); E. J. Galvez, B. E. Sauer, L. Moorman, P. M. Koch and D. Richards, Phys. Rev. Lett. 61, 2011 (1988).

[12] Another example is the ion in a Paul trap interacting with a standing light field. The ion is driven either by the explicit time dependence of the trap or of the standing wave. See for example, M. El Ghafar, P. Törmä, V. Savichev, E. Mayr, A. Zeiler and W. P. Schleich, Phys. Rev. Lett. 78, 4181 (1997); S. A. Gardiner, J. I. Cirac and P. Zoller, Phys. Rev. Lett. 79, 4790 (1997); S. A. Gardiner, J. I. Cirac and P. Zoller, Phys. Rev. Lett. 80, 2968 (1998); G. M. Zaslavsky, Chaos in Driven Systems (Harwood, Chur, 1985).

[13] In many systems such as the kicked rotor or the atom in the phase modulated light wave there is dynamical localization only in the momentum variable. See for example, P. J. Bardroff, I. Bialynicki-Birula, D. S. Krähmer, G. Kurizki, E. Mayr, P. Stifter and W. P. Schleich, Phys. Rev. Lett. 74, 3959 (1995).

[14] We indicate the physical variables with tildas and we will drop all the tildas later when we introduce the dimensionless variables.

[15] C. Aminoff, A Steane, P. Bouyer, P. Desbiolles, J. Dalibard and C. Cohen-Tannoudji, Phys. Rev. Lett. 71, 3083 (1993).

[16] This value of $k$ is also consistent with the commutation relation $[z, p]=[\tilde{z}, \tilde{p}] \omega^{3} /\left(m g^{2}\right)=i \hbar \omega^{3} /\left(m g^{2}\right) \equiv i \hbar$ for the dimensionless canonical variables $z$ and $p$.

[17] N. Brenner and S. Fishman, Phys. Rev. Lett. 77, 3763 (1996).

[18] B.V. Chirikov, Phys. Rep. 52, 263 (1979).

[19] C. Tsallis, Physics World 10(7), 42 (1997).

[20] See for example, C. Leichtle, I. Sh. Averbukh and W. P. Schleich, Phys. Rev. Lett. 77, 3999 (1996); Phys. Rev. A 54, 5299 (1996).

[21] F. Saif, G. Alber, V. Savichev and W. P. Schleich (to be published).

[22] Yu. B. Ovchinnikov, I. Manek and R. Grimm, Phys. Rev. Lett. 79, 2225 (1997).

[23] T. M. Roach, H. Abele, M. G. Boshier, H. L. Grossman, K. P. Zetie and E. A. Hinds, Phys. Rev. Lett. 75, 629 (1995). 

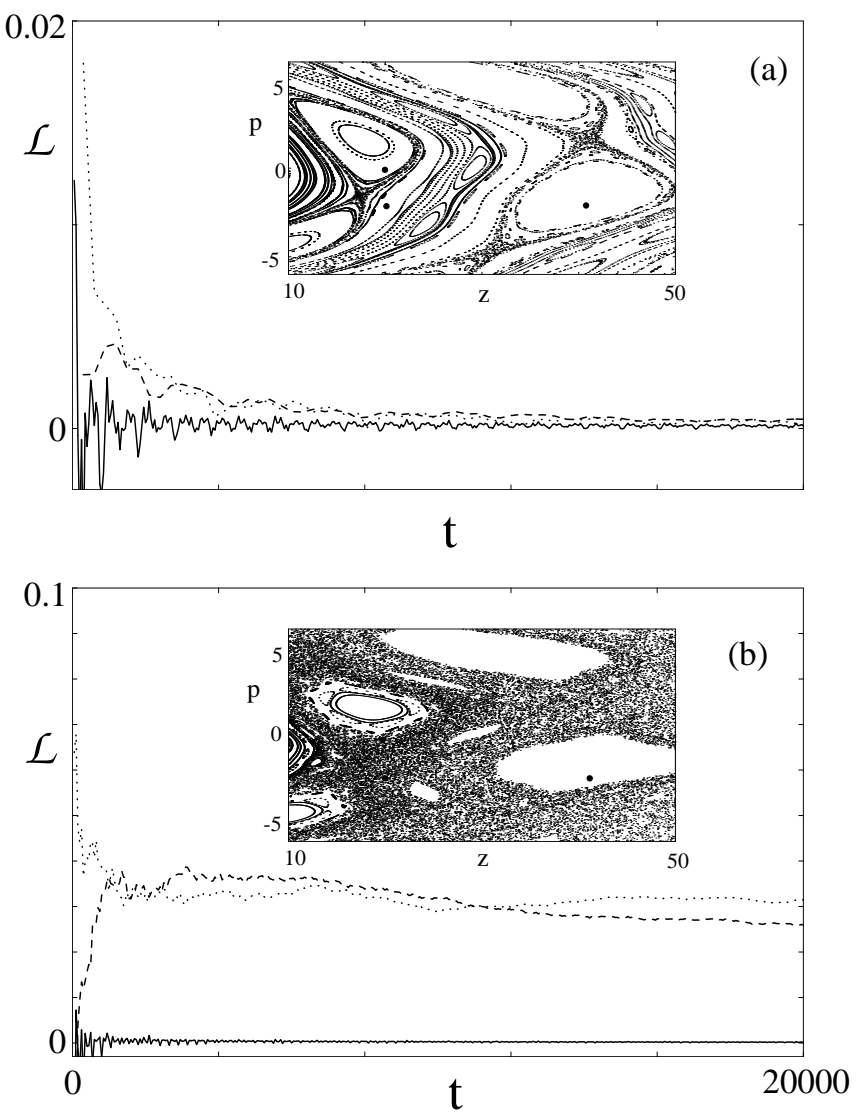

FIG. 1. Lyapunov exponent $\mathcal{L}$ of an atomic Fermi accelerator below (a) and above (b) the lower boundary $\lambda_{l}=0.24$ of the localization window. We use three initial conditions $\left(z_{0}, p_{0}\right)=\{(20,0) ;(20,-2) ;(40,-2)\}$ represented by thick dots in the Poincaré sections. In (a) we have chosen the modulation depth $\lambda=0.2$ and all initial conditions lie inside isolated resonances. Consequently for all three initial conditions the Lyapunov exponent approaches zero. In (b) we have $\lambda=0.5>\lambda_{l}$. Here, the phase space point $(40,-2)$ still sits in an island whereas the points $(20,0)$ and $(20,-2)$ lie in the stochastic sea. As a result the Lyapunov exponent for the first initial condition converges to zero whereas for the other two it is positive. Here and in all other figures we have chosen for the height of the potential $V_{0}=60$ and for its steepness $\kappa=0.5$. 

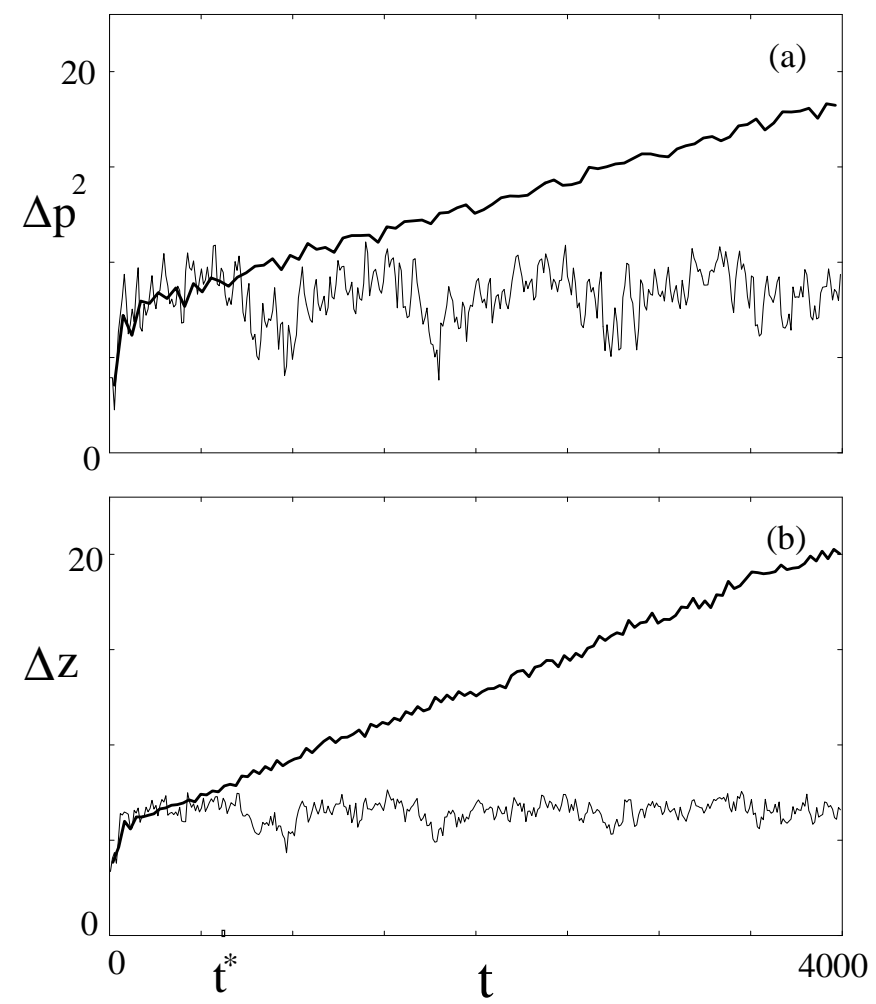

FIG. 2. Comparison between quantum (thin lines) and classical (thick lines) values of the (a) square of the width $\Delta p$ of the momentum distribution and (b) width of the position distribution $\Delta z$. In the quantum case we integrate the Schrödinger equation, Eq. (4), subjected to the initial condition of a Gaussian minimum uncertainty wavepacket located at the phase space point $(20,0)$ with a width $\Delta z=2$ in position and the corresponding width $\Delta p=\hbar /(2 \Delta z)$ in momentum. In the classical case we propagate an ensemble of particles distributed according to the same Gaussian distribution using the classical Hamilton equations, Eq. (3). The number of particles in the classical simulation is 2000. The height of the exponential potential is $V_{0}=60$, its steepness $\kappa=0.5$ and the modulation strength is $\lambda=0.5$. In the quantum mechanical case the effective Planck's constant is taken as $k=4$. 

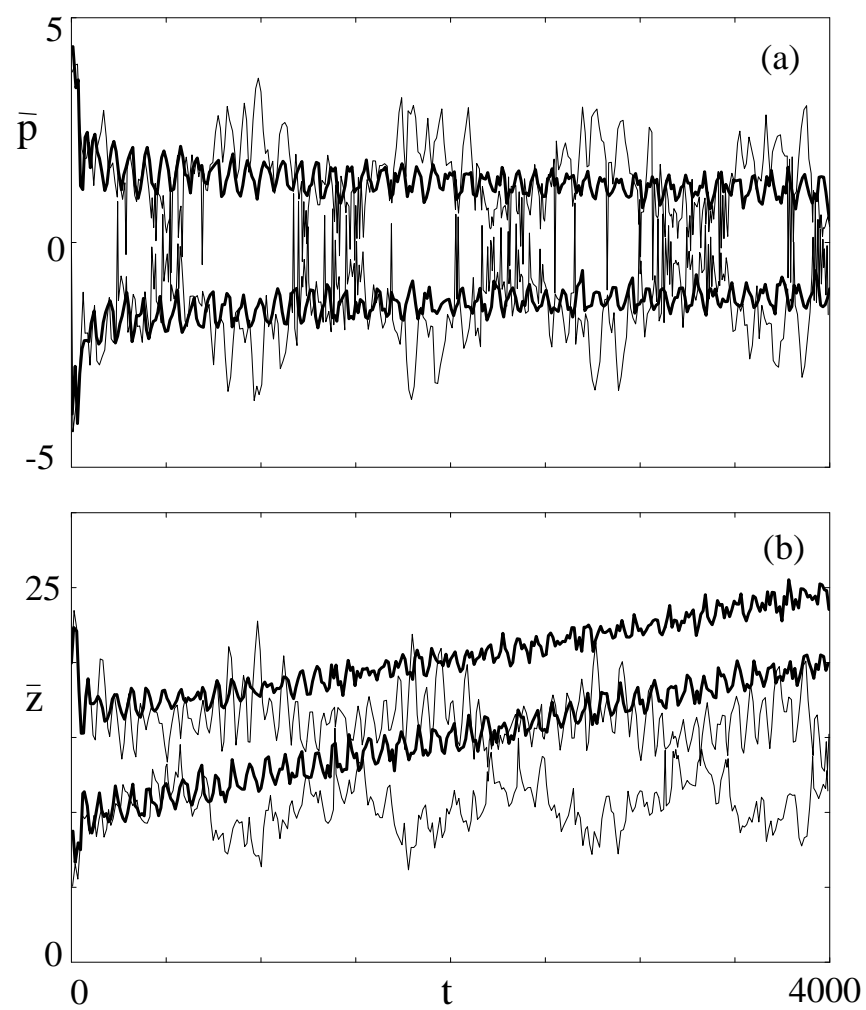

FIG. 3. Comparison between the classical (thick lines) and quantum (thin lines) average momentum (a) and average position (b) as functions of time. For the sake of presentation, we show the envelope of the corresponding functions. We note the modulation of the quantum mechanical envelopes which is absent in the classical curves. This is a manifestation of revivals 20 in a driven quantum system [21. The calculations were performed for the same set of parameters as in Fig. 2 . 

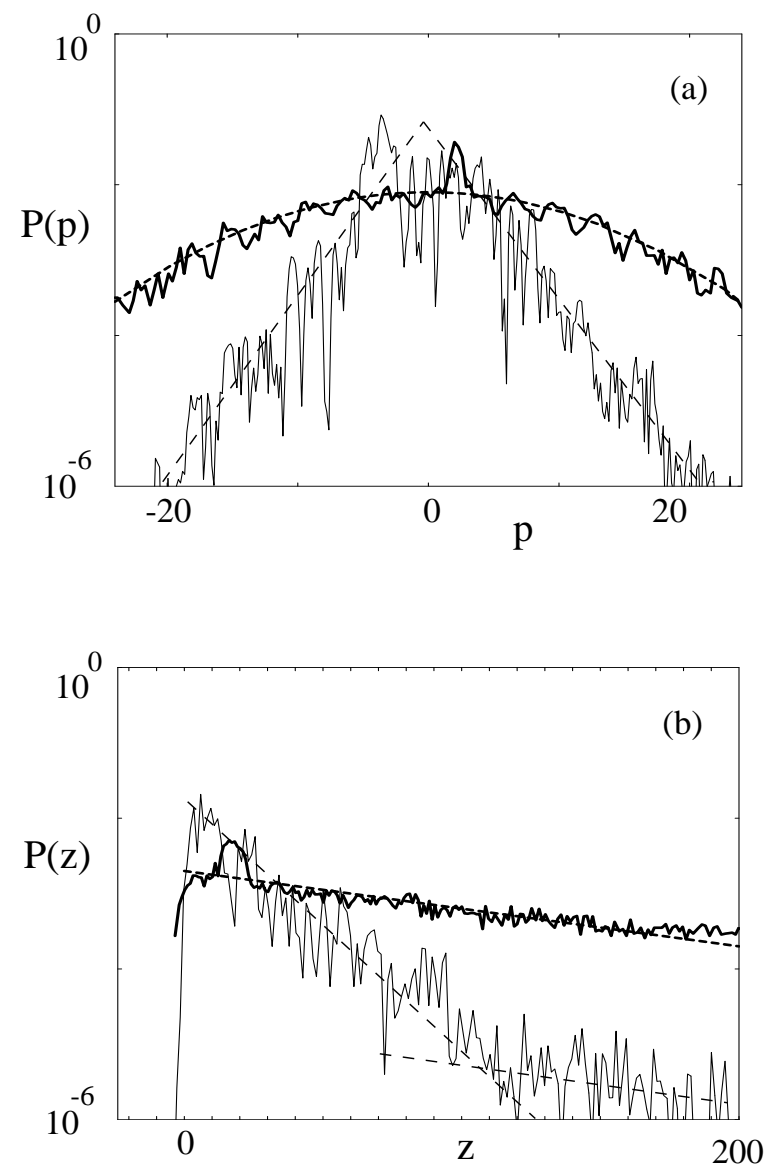

FIG. 4. Comparison between the quantum mechanical (thin lines) and classical (thick lines) momentum (a) and position (b) distributions on a logarithmic scale for an atomic Fermi accelerator. The momentum distribution in the quantum mechanical case exhibits an exponential localization, whereas the corresponding classical distribution is Gaussian. The classical as well as the quantum mechanical position distributions are both of exponential form. However in the classical case this form results from the linear potential in the Boltzmann distribution. The peak around $z=20$ is due to the fact that a considerable part of our initial ensemble lies inside a stable island. In the quantum case we find two exponentials: the flat one is a remnant of the classical Boltzmann distribution and the steeper one represents dynamical localization. The dashed lines indicate linear and quadratic fits which correspond to exponential and Gaussian distributions, respectively. Here we have chosen $\lambda=0.8$ and the number of particles in the classical simulation is 10000. The integration time is $t=2650$ and all the other parameters are the same as in Fig 2 .

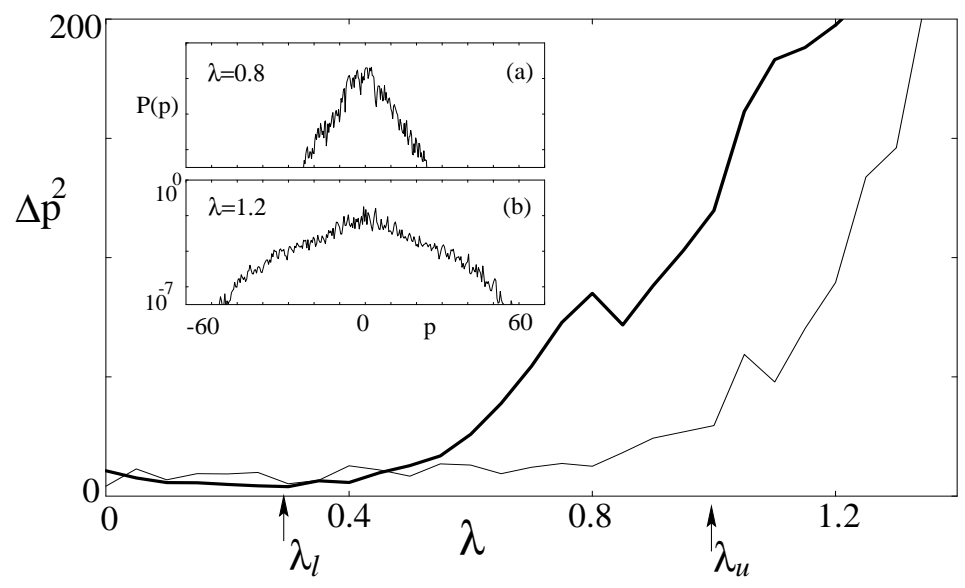


FIG. 5. Localization window defined by the onset of classical and quantum diffusion at $\lambda_{l}=0.24$ and $\lambda_{u}=\sqrt{k} / 2=1$, respectively. Squares of the width of the classical (thick line) and the quantum mechanical (thin line) momentum distributions in their dependence on $\lambda$. The two curves start to separate at $\lambda=\lambda_{l}$. A transition from a localized to a delocalized quantum mechanical momentum distribution occurs at $\lambda_{u}$. For $\lambda=0.8$ which for $k=4$ lies well within the window we find exponential localization [inset (a)]. In contrast for $\lambda=1.2$ which lies outside of the window we find a broad Gaussian distribution indicating delocalization [inset (b)]. All the other parameters are as in Fig. 2 and $t=3200$. 